ORIGINAL ARTICLE

\title{
High bone mineral density in loaded skeletal regions of former professional football (soccer) players: what is the effect of time after active career?
}

\author{
K Uzunca, M Birtane, G Durmus-Altun, F Ustun
}

Br J Sports Med 2005;39:154-158. doi: 10.1136/bjsm.2003.011494

See end of article for authors' affiliations

Correspondence to: Assistant

Professor Kaan Uzunca, Trakya University, Medical Faculty, Physical Medicine and Rehabilitation, Istasyon Mah, Istasyon Cad, Istasyon Apt, Daire 8, Edirne 22030, Turkey; druzunca@yahoo.com

Accepted 8 June 2004

\begin{abstract}
Objectives: Physical exercise is an important factor in the acceleration and maintenance of bone mineral density (BMD). Football is an impact loading sport and some studies demonstrate its site specific, bone mass increasing effect. We compared BMD at different skeletal regions in a group of former professional football players and in normal control subjects and evaluated the effect of demographic factors and time after active career on BMD.

Methods: Twenty four former football players $<70$ years old who had retired from professional football at least 10 years previously and 25 non-athletic controls were recruited. The demographic characteristics, activity levels, and dietary habits of all subjects and the chronological history of the footballers' professional careers were noted. BMD was measured by DEXA at the calcaneus and distal tibia and at the lumbar spine, proximal femur, and distal and proximal radius, and compared between groups. Stepwise multiple linear regression analysis was used to determine the probable predictors of BMD in former football players.

Results: In former players BMD values were found to be significantly higher at the lumbar spine, femur neck, femur trochanter, distal tibia, and calcaneus, but not at Ward's triangle (femur) or the distal and proximal radius regions compared with controls. Time after active career was the only independent predictor of BMD at the lumbar spine, proximal femur (neck, trochanter, and Ward's triangle), and distal tibia.

Conclusions: Former footballers had higher BMD at weight loaded sites and time after active career seemed to be an important factor in determining BMD.
\end{abstract}

t is commonly accepted that physical exercise and sports training are important factors in the acceleration and maintenance of bone mineral density (BMD). The mechanism of the beneficial effect of exercise on bone metabolism has been the focus of intensive research. Many studies reported that BMD increases in athletes were mainly due to the strain resulting from impact bone loading, rather than and independent from muscle strength increases. ${ }^{12}$ It has been reported that athletes who participate in impact loading sports, such as tennis, volleyball, and gymnastics, have higher BMD, ${ }^{1-6}$ while swimmers and cyclists who practice unloaded and partly unloaded sports, respectively, do not, when compared with non-athletes. ${ }^{4}$ Some reports even suggested that swimmers had lower BMD than non-athletic controls. ${ }^{8}$ Exercise seem to have a beneficial effect on bone by stimulating the osteogenic responses according to some studies. ${ }^{9-11}$

Football is probably the most popular sport among the male population in Turkey and is characterised by various types of running with rapid changes of direction, starts, stops, jumping, and kicking resulting in large ground reaction forces at the skeleton, and thus can be classified as an impact loading sport. ${ }^{2}$ Some studies demonstrate the site specific, bone mass increasing effect of football which bears significant weight on specific skeletal regions. ${ }^{2} 10{ }^{12}$ However, only a few studies, mainly by Swedish researchers, have investigated how the status of this increased BMD changes in the years after the end of an active football career. ${ }^{13}{ }^{14} \mathrm{We}$, therefore, sought to examine this issue by comparing a group of professional football players who had retired at least 10 years previously with non-athletic subjects in terms of $\mathrm{BMD}$ in different skeletal regions such as the calcaneus and distal tibia in addition to standard sites of measurement such as the lumbar spine, proximal femur, and distal and proximal radius.

\section{METHODS}

This study was approved by a local ethics committee.

\section{Subjects}

The group of footballers consisted of 24 former players below the age of 70 who had retired from their careers in the second professional football league at least 10 years previously. Professional football players in particular were selected as they were considered to have undergone standardised intensive training and match programmes. A professional football player usually practices for a mean of 12-14 h a week (including match activity) for 40 weeks a year. We also included 25 non-athletic volunteer controls from among our hospital employees, with no history of an active sports career and matched for demographic characteristics. The former footballers or control subjects were not included in the study if they had: (i) a secondary disease leading to bone loss, such as hypogonadism, hyperthyroidism, hyperparathyroidism, multiple myeloma, diabetes, etc; (ii) any medical condition such as cardiovascular diseases or inflammatory or degenerative arthritis leading to immobility; or (iii) a history of drug therapy known to effect bone metabolism and including the administration of glucocorticosteroids, methotrexate, diuretics, heparin, anticonvulsants, etc. None of the former

Abbreviations: $B M D$, bone mineral density; $B M I$, body mass index; $\mathrm{Cl}$, confidence interval; CV, coefficient of variation; DEXA, dual energy $x$ ray absorptiometer; ROI, regions of interest 
players smoked so we selected control subjects from nonsmokers. The demographic characteristics of all study participants, such as age, weight, height, and body mass index (BMI), were noted. The age of starting their professional career, the length of the career, the reasons for retiring, and number of years since the retirement of the former professional football players were noted.

\section{Life style evaluation}

The current activity levels of all subjects were classified as follows: (1) no sporting activity, sedentary life style; (2) no sporting activity, active life style; (3) sporting activity once a month; (4) sporting activity once a week; and (5) regular sporting activity at least three times a week. Subjects in a sedentary job and having their daily needs attended to by someone else were determined as having a sedentary life style, while those with an active workload and attending to their own daily needs were determined as having an active life style. The sports activities considered were non-professional weight bearing activities such as amateur football, tennis, walking, jogging, etc.

Nutritional habits such as diet, alcohol consumption, and coffee intake were noted. The daily consumption of calcium of all study participants was approximately calculated according to the intake of dairy products with known quantities of calcium. ${ }^{15}$

\section{Dual energy $x$ ray absorptiometer (DEXA) measurement}

Areal bone density was measured using a DEXA instrument (Norland XR36, Norland Medical Systems, Fort Atkinson, WI, USA). Distal and proximal radius, lumbar spine (anteroposterior and lateral, L2-L4), and proximal femur (hip) scans were performed according to the manufacturer's procedures. All scans, except for those in the lumbar spine region, were performed at the dominant extremity. Additional imaging was acquired in research mode and analysis was applied the distal tibia and calcaneus using the standardised regions of interest (ROI) method (host software version: Norland 3.9.4). A rectangular shaped ROI with an area of $\sim 3 \mathrm{~cm}^{2}$ was chosen in the trabecular bone region of the distal tibia and calcaneus. For statistical analysis, BMD values $\left(\mathrm{g} / \mathrm{cm}^{2}\right)$ of all ROIs were obtained. In our laboratory, the coefficient of variation (CV) values for BMD were estimated to be $1.0 \%$ (lumbar L2-4 spine), $1.2 \%$ (femoral neck), $1.8 \%$ (femur trochanter), 3.7\% (femur Ward's triangle), $0.8 \%$ (proximal radius), $0.8 \%$ (distal radius), $1.5 \%$ (distal tibia), and $2.2 \%$ (calcaneus).

\section{Statistical analysis}

Student's $t$ tests for independent samples were employed to compare patient characteristics, daily calcium intake, and BMD values between the two groups. The group comparisons for current activity levels, alcohol, and coffee consumption were performed using $\chi^{2}$ test. Univariate correlation analysis was performed using Pearson's correlation test to assess the relation between age, BMI, calcium intake, and the BMD of various bone regions in all subjects. Also correlation between time after active career and BMD of the measurement sites in former football players was assessed by Pearson's correlation analysis. Stepwise multiple linear regression analysis was used to determine the probable predictors of BMD at all measurement regions in former professional football players. Here, the dependant variable was BMD in all regression models. All regression models contained time after active sports career with one more (age) and two more (age and BMI) independent variables.

\section{RESULTS}

The 24 male former football players subjects had an average age of 52 (min-max: 40-68) while 25 subjects in the control group had an average age of 54 (min-max: 39-69). The groups were comparable in terms of mean age, height, weight, and BMI (table 1). The mean (standard deviation, SD) age of starting their professional football career in former players was 16.5 (2.3) years. The mean length of the professional career was 16.5 (4.5) years and the mean number of years since retirement was 19.3 (7.9) years. All the former players had retired from active career because of ageing except for two, one of whom had retired because of a bone fracture and the other because of a meniscus injury. However, none of the former players had inflammatory or degenerative arthritis causing immobility.

The activity levels (see Life style evaluation section) of the former footballers were 1 (five), 2 (three), 3 (four), 4 (six), and 5 (six), while in the control group there were five subjects at each activity level. Current activity levels were not statistically different between the groups $(p>0.05)$ nor did the groups have different diets. All participants consumed normal amounts of meat (3-5 days a week) and none were vegetarian. The proportion of alcohol consumers did not differ between the groups $(83.3 \%$ in former players $v 80 \%$ in control subjects, $\mathrm{p}>0.05$ ) and high daily consumption rates below alcoholism limits were not different $(4.17 \% \vee 4 \%$, respectively, $\mathrm{p}>0.05)$. Each day $41.6 \%$ of former football players and $40 \%$ of control subjects consumed at least one cup of traditionally prepared coffee, with a non-significant difference $(\mathrm{p}>0.05)$. The mean (SD) daily calcium intake was not different between the two groups (727 (251) v 735 (240) $\mathrm{mg}$ ) in former players and control subjects respectively, $\mathrm{p}>0.05$ ).

BMD values were found to be significantly higher in former players at the lumbar spine, femur neck, femur trochanter, distal tibia and calcaneus when compared with control subjects. However, no statistically significant difference was detected at femur Ward's triangle or the distal and proximal regions of the radius (table 1).

According to correlation analysis, age correlated significantly with femur neck BMD $(r=-0.36, \mathrm{p}<0.01)$ and femur Ward's triangle BMD $(r=-0.47, \mathrm{p}<0.001)$ in all subjects. BMI correlated significantly with distal radius BMD $(r=0.44$, $\mathrm{p}<0.01)$ and distal tibia BMD $(r=0.35, \mathrm{p}<0.05)$ in all participants. No such correlation was found between daily calcium intake and BMD of any measurement region. Correlation analysis revealed significant correlation with time after active career and lumbar spine BMD $(r=-0.44$, $\mathrm{p}<0.05)$, femur neck BMD $(r=-0.55, \mathrm{p}<0.01)$, femur trochanter BMD $(r=-0.48, \mathrm{p}<0.05)$, and femur Ward's triangle $\operatorname{BMD}(r=-0.65, \mathrm{p}<0.001)$ in former football players.

When the potential of time after active career, age, and $\mathrm{BMI}$ to predict the BMD values at the various measurement regions of former footballers was assessed by stepwise multiple linear regression analysis, time after active career was found to be the single independent predictor of BMD at the lumbar spine (fig 1), proximal femur (neck, trochanter, and Ward's triangle), and distal tibia with the same constant in all regression models containing one more (age) and two more independent variables (age and BMI) in addition to time after active career. The regression equations are given in table 2. No such relation was detected at the proximal-distal radius or calcaneus.

\section{DISCUSSION}

Higher bone mass is expected in the loaded skeletal regions of athletes playing impact loading sports. Previous studies have demonstrated higher BMD in the dominant upper extremity 
Table 1 Comparison of characteristics and BMD of the groups

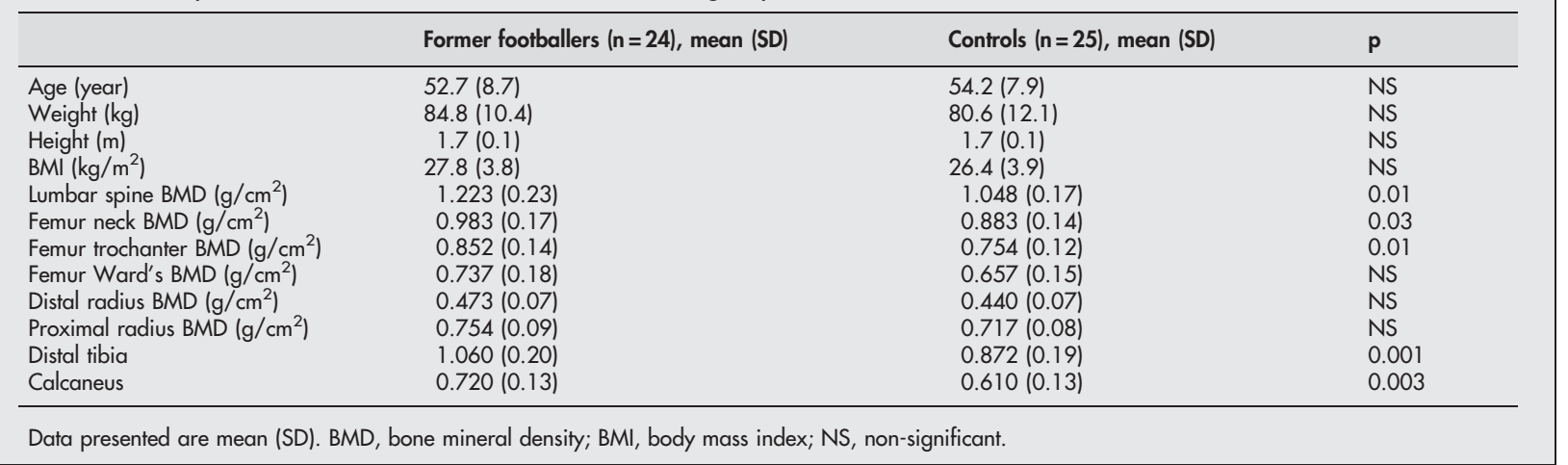

bones of tennis players. ${ }^{6}{ }^{16}$ Likewise, volleyball players were shown to have higher bone mass in their smashing arms compared with their non-dominant arms. ${ }^{13}$ Studies investigating the BMD of footballers have been performed mainly using female subjects, however recently some reports on male subjects have been published. ${ }^{14} 1{ }^{17}{ }^{18}$ In previous studies the impact of time after active sports on the BMD in loaded regions, the focus of this study, have not been investigated in detail.

It is generally difficult, in such BMD evaluating studies, to recruit similar groups of patients, since bone metabolism is influenced by many factors. We found the usual measurable demographic parameters (age, BMI, nutrition conditions) comparable between the groups, but it was difficult to standardise the activity levels. We did not classify the subjects according to their duties or social classes, but according to their actual life activities. Subjects with medical conditions affecting bone metabolism or leading to immobility were not included in the study.

Only former male footballers were selected as it would be difficult to evaluate the probable confounding effect in female subjects of strenuous exercise induced menstruation disorders and amenorrhea which can reduce bone mass and to some extent negate the effect of exercise on bones. ${ }^{19}{ }^{20}$ Although it was shown that strenuous exercise negatively correlated with serum testosterone, the levels remained within the normal range. ${ }^{21}$

We assessed BMD not only at the standard DEXA measurement sites (proximal femur, lumbar spine, and distal and proximal radius), but also at the sites which we consider to be loaded significantly during football, such as the distal tibia and calcaneus which were not widely evaluated previously. We only measured the dominant extremities which were previously shown to be more affected.

In this study former footballers who had retired from professional football at least 10 years previously were observed to have higher BMD at the lumbar spine, proximal femur (neck, trochanter, and Ward's triangle), distal tibia, and calcaneus when compared with control subjects; however, no such superiority was detected at the proximal or distal radius of dominant extremities. The discrepancy we found at various regions is not surprising according to Karlsson's hypothesis of reciprocal change in BMD between unloaded and loaded skeletal regions. ${ }^{22}$ This hypothesis claims that the weight loaded regions impacted during a specific sport should have higher BMD while the partly loaded regions have equal BMD and unloaded regions have lower BMD when compared with controls. Our findings were in accordance with this hypothesis as we did not find significant differences at the proximal and distal radius which are partly loaded regions according to Magnusson et al. ${ }^{13}$ We did not compare the unloaded sites as it was impossible because football loads every bone including the skull.

The lower extremities of football players are exposed to high ground reaction forces in a range of three to six times the body weight during activity. ${ }^{23}$ It is also known that the osteogenic response of loaded bones is related not only to the magnitude but also to the direction and the characteristic of the load. Data obtained from experimental studies suggest that load bearing most probably exerts its influence through the dynamic strains engendered in bone tissue. ${ }^{11}$ Unusual strain distributions, high strains, and high strain rates seem to be particularly osteogenic. ${ }^{24}$ Alfredson et $a l^{2}$ claimed that the diaphysis of femur is particularly subjected to compressive forces while the hip (femur neck, trochanter, and Ward's triangle) and knee regions are especially loaded both with compressive and shear forces. The higher BMD we found in our former football players not only at the hip region but also at ankle and foot bones (distal tibia and calcaneus) is not surprising as these sites consist mainly of trabecular bone and are subjected to multidirectional, dynamic compressive, and shear forces during football activity. ${ }^{25}$ Proximal femur, distal tibia and calcaneus bones mainly contain trabecular bone which has a higher metabolic rate than cortical bone and reacts more to the applied strains. ${ }^{26}$ However, as we did not measure any cortical bone in this study we are unable to compare or draw strong conclusions on this point.

Karlsson $e t$ al $^{27}$ investigated whether the beneficial effect of weight lifting on BMD was maintained in the long term in former weight lifters. The authors reported that BMD values

Table 2 Regression equations predicting BMD

\begin{tabular}{|c|c|c|}
\hline \multirow[b]{2}{*}{ Equation } & \multicolumn{2}{|l|}{$95 \% \mathrm{Cl}$} \\
\hline & Min & Max \\
\hline $\begin{array}{l}\text { Lumbar spine BMD }\left(\mathrm{g} / \mathrm{cm}^{2}\right)=1.472-(0.01 \times \text { years after active career) } \\
\text { Femur neck BMD }\left(\mathrm{g} / \mathrm{cm}^{2}\right)=1.216-(0.01 \times \text { years after active career) } \\
\text { Femur trochanter } B M D\left(\mathrm{~g} / \mathrm{cm}^{2}\right)=1.013-(0.009 \times \text { years after active career) } \\
\text { Femur Ward's triangle BMD }\left(\mathrm{g} / \mathrm{cm}^{2}\right)=0.999-(0.01 \times \text { years after active career) } \\
\text { Distal tibia BMD }\left(\mathrm{g} / \mathrm{cm}^{2}\right)=1.251-(0.01 \times \text { years after active career) }\end{array}$ & $\begin{array}{l}-0.026 \\
-0.021 \\
-0.016 \\
-0.023 \\
-0.021\end{array}$ & $\begin{array}{l}-0.002 \\
-0.006 \\
-0.002 \\
-0.007 \\
-0.001\end{array}$ \\
\hline
\end{tabular}




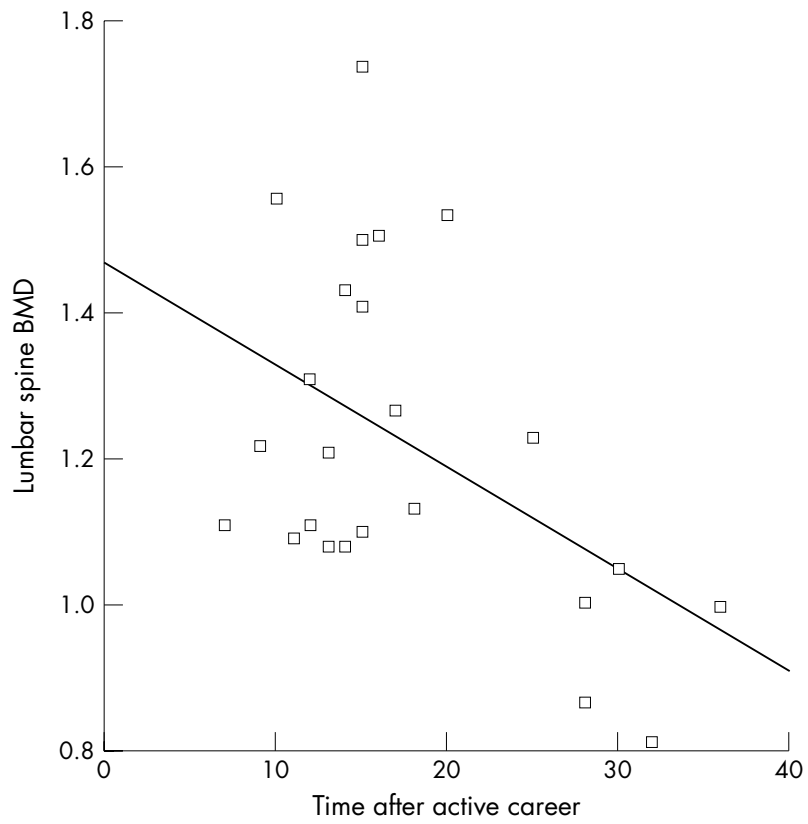

Figure 1 Relationship between time after active career and lumbar spine BMD.

were significantly higher in former athletes aged 50-64 compared with control subjects, but no such favourable findings were observed in the study population above 65 years of age. Only a few studies report data on the process of BMD change in former footballers. According to the results of two studies, football players subjected to impact loading in their early adulthood had higher BMD values when they were older. ${ }^{12}{ }^{13}$ Magnusson et $a l^{13}$ stated that no BMD superiority remained compared with controls after 70 years of age. In this study we showed by linear regression analysis that time after active career is the only independent predictor of BMD in our former footballers with a mean age of 52 years, while unexpectedly no such correlation was found for age and BMI. We did not include former players above the age of 70 to exclude the adverse effect of senility on bones; this might be the main reason why we did not find any effect of age on BMD.

In conclusion, our study showed that time after active career predicts BMD in former players. However, the number of subjects studied was too few to determine a cut off value defining the end point of the BMD advantage when compared with control subjects. Also a larger sample size with more demographic independent variables would have been better for regression analysis and would have allowed stronger conclusions. This may be why we could not find an effect of age or BMI on BMD. Another limitation was that, except for the lumbar spine, we only could assess BMD in specific ROI sites with a limited area. Therefore, we could not evaluate bone size variations between groups. A study with a larger population and taking bone size variations into account would provide more sophisticated findings.

\section{What is already known on this topic}

Football is an impact loading sport affecting specific bones. It is known that these bones have higher BMD than non-weight bearing skeletal regions. However, this bone advantage diminishes with time following retirement from professional football.
What this study adds

This study was the first to evaluate the effect of time after retirement on the BMD of former footballers and shows that time after active career predicts BMD in former players.

\section{Authors' affiliations}

K Uzunca, M Birtane, Physical Medicine and Rehabilitation Department, Medical Faculty, Trakya University, Edirne, Turkey

G Durmus-Altun, F Ustun, Nuclear Medicine Department, Medical Faculty, Trakya University, Edirne, Turkey

Competing interests: none declared

\section{REFERENCES}

1 Alfredson $\mathbf{H}$, Nordstrom P, Pietila T, et al. Long-term loading and regional bone mass of the arm in female volleyball players. Calcif Tissue Int 1998;62(4):303-8.

2 Alfredson $\mathrm{H}$, Nordstrom P, Lorentzon R. Total and regional bone mass in female soccer players. Calcif Tissue Int 1996;59(6):438-42.

3 Calbet JA, Diaz Herrera P, Rodriguez LP, et al. High bone mineral density in male elite professional volleyball players. Osteoporos Int 1999;10(6):468-74.

4 Fehling PC, Alekel L, Clasey J, et al. A comparison of bone mineral densities among female athletes in impact loading and active loading sports. Bone 1995;17(3):205-10.

5 Kontulainen S, Kannus P, Haapasalo H, et al. Good maintenance of exerciseinduced bone gain with decreased training of female tennis and squash players: a prospective 5-year follow-up study of young and old starters and controls. J Bone Miner Res 2001;16(2):195-201.

6 Calbet JA, Moysi JS, Dorado C, et al. Bone mineral content and density in professional tennis players. Calcif Tissue Int 1998;62(6):491-6.

7 Heinonen A, Oja P, Kannus P, et al. Bone mineral density of female athletes in different sports. Bone Miner 1993;23(1): 1-14.

8 Taaffe DR, Snow-Harter C, Connolly DA, et al. Differential effects of swimming versus weight-bearing activity on bone mineral status of eumenorrheic athletes. J Bone Miner Res 1995; 10(4):586-93.

9 Rutherford OM. Is there a role for exercise in the prevention of osteoporotic fractures? Br J Sports Med 1999;33(6):378-86.

10 Soderman $\mathrm{K}$, Bergstrom $\mathrm{E}$, Lorentzon R, et al. Bone mass and muscle strength in young female soccer players. Calcif Tissue Int 2000;67(4):297-303.

11 Lanyon LE. Control of bone architecture by functional load bearing. $J$ Bone Miner Res 1992;7(suppl 2):S369-75.

12 Duppe H, Gardsell P, Johnell O, et al. Bone mineral density in female junior, senior and former football players. Osteoporos Int 1996;6(6):437-41.

13 Magnusson $\mathrm{H}$, Linden $\mathrm{C}$, Karlsson C, et al. Exercise may induce reversible low bone mass in unloaded and high bone mass in weight-loaded skeletal regions. Osteoporos Int 2001;12(11):950-5.

14 Karlsson MK, Linden C, Karlsson C, et al. Exercise during growth and the bone mineral density and fractures in old age. Lancet 2000;355(5):469-70.

15 Nordin C. Calcium and osteoporosis. Nutrition 1997;13(7-8):664-86.

16 Kontulainen S, Sievanen $\mathrm{H}$, Kannus $\mathrm{P}$, et al. Effect of long-term impactloading on mass, size, and estimated strength of humerus and radius of female racquet-sports players: a peripheral quantitative computed tomography study between young and old starters and controls. J Bone Miner Res 2002;17(12):2281-9.

17 Vicente-Rodriguez G, Jimenez-Ramirez J, Ara I, et al. Enhanced bone mass and physical fitness in prepubescent footballers. Bone 2003;33:853-9.

18 Calbet JAL, Dorado C, Díaz-Herrera P, et al. High femoral bone mineral content and density in male football (soccer) players. Med Sci Sports Exerc 2001;33(10): 1682-7.

19 Rencken ML, Chesnut CH 3rd, Drinkwater BL. one density at multiple skeletal sites in amenorrheic athletes. JAMA 1996;276(3):238-40.

20 Nattiv A. Stress fractures and bone health in track and field athletes. J Sci Med Sport 2000;3(3):268-79.

21 MacKelvie KJ, Taunton JE, McKay HA, et al. Exercise may induce reversible low bone mass in unloaded and high bone mass in weight-loaded skeletal regions. Osteoporos Int 2001;12(11):950-5.

22 Karlson MK, Hasserius R, Obrant KJ. Bone mineral density in athletes during and after career: a comparison between loaded and unloaded skeletal regions. Calcif Tissue Int 1996;59:245-8.

23 Giddings VL, Beaupre GS, Whalen RT, et al. Calcaneal loading during walking and running. Med Sci Sports Exerc 2000;32(3):627-34.

24 Lanyon LE. Using functional loading to influence bone mass and architecture: objectives, mechanisms, and relationship with estrogen of the mechanically adaptive process in bone. Bone 1996:18(1 suppl):37-43.

25 Scott SH, Winter DA. Internal forces of chronic running injury sites. Med Sci Sports Exerc 1990;22(3):357-69

26 Prevharl S, Fuerst T, Fan B, et al. Quantitative ultrasound of the tibia depends on both cortical density and thickness. Osteoporos Int 2001;12:28-34.

27 Karlsson MK, Johnell O, Obrant KJ. Is bone mineral density advantage maintained long-term in previous weight lifters? Calcif Tissue Int 1995;57(5):325-8. 
This paper evaluates the Achilles heel of exercise-its cessation. Exercise during growth enhances bone mineral density (BMD), but what happen with retirement from activity? The current study indicates a higher BMD loss with retirement, these data supporting previous publications which evaluated retired ballet dancers and male soccer players also above the age of 65 ; these papers report that no BMD benefits were found after this age. In addition, prospective studies following athletes in retirement indicate a higher BMD loss compared to both athletes who continue with exercise and controls. In spite of this, there exist data indicating that former athletes have fewer fragility fractures than controls. The reason for this lower fracture incidence is unclear, but remaining exercise induced benefits in skeletal architecture, bone size, muscle function, or balance may explain the findings as well as a selection bias at baseline, so that physically fit individuals both choose to exercise and have fewer fractures.

M K Karlsson

Department of Orthopaedics, Lund University, Malmo General Hospital, Malmo SE-205 02, Sweden; magnus.karlsson@orto.mas.lu.se

\section{ELECTRONIC PAGES}

\section{Online original articles}

7 he following electronic only articles are published in conjunction with this issue of BJSM (see also pages 136 and 165)

\section{Proprioceptive neuromuscular facilitation training induced alterations in muscle fibre type and cross sectional area \\ N Kofotolis, I S Vrabas, E Vamvakoudis, et al}

Objectives: To compare the effects of proprioceptive neuromuscular facilitation (PNF) and isokinetic training on fibre type distribution and cross sectional area of the vastus lateralis muscle.

Methods: Twenty four male university students were divided into two equal groups: PNF training and isokinetic training (ISO). The training regimen for the PNF group consisted of three sets of 30 repetitions against maximal resistance, alternating two patterns of sequential movements of the right lower extremity: $(a)$ toe flexion and ankle plantar flexion and eversion; $(b)$ knee extension and hip extension, abduction, and internal rotation. The ISO group performed three sets of 30 repetitions alternating knee extension and flexion of the right leg at angular velocities of 180 and $90 \% \mathrm{~s}$ in an isokinetic dynamometer (Cybex). Both groups trained three times a week for a total of eight weeks. Muscle biopsy specimens were obtained from the right vastus lateralis muscle before and after training.

Results: The mean percentage area of type IIB fibre was significantly decreased $(\mathrm{p}<0.01)$ after eight weeks of PNF training, whereas that of type IIA fibre was significantly $(p<0.05)$ increased. The mean percentage area of ISO trained type IIAB fibres exhibited an augmentative pattern $(\mathrm{p}<0.01)$ with a parallel reduction $(\mathrm{p}<0.05)$ in type IIA. Percentage fibre type distribution exhibited a similar pattern.

Conclusions: Both PNF and ISO training alter fibre type distribution and mean cross sectional area. These changes occur in the type II fibre subgroup.

(Br J Sports Med 2005;39:ell) http://bjsm.bmjjournals.com/ cgi/content/full/39/3/ell

\section{Physical activity levels during phase IV cardiac rehabilitation in a group of male myocardial infarction patients K Woolf-May, S Bird}

Objective: To determine physical activity levels during phase IV cardiac rehabilitation in 31 male myocardial infarction patients (median age 62, range 53-77 years).

Methods: Patients recorded daily physical activity over 16 weeks in a diary. Diaries were analysed for total general physical activity (TGPA), leisure time physical activity (LTPA), and "active for life" exercise classes (AFL). Pre- and post-observation period (OP) subjects underwent a $10 \mathrm{~m}$ shuttle walking test (SWT) to determine changes in aerobic fitness. Rate of perceived exertion (RPE) determined exercise intensity. Estimated gross energy expenditure (EEE) was determined by a regression equation between RPE and $\mathrm{VO}_{2}\left(1 \mathrm{~min}^{-1}\right.$ during SWT. A total of $97 \%$ of subjects were on lipid lowering medication.

Results: There were no correlations between $\mathrm{Vo}_{2}\left(\mathrm{l} \mathrm{min}^{-1}\right)$ and body mass, therefore $\mathrm{kcal} \mathrm{min}^{-1}$ indicated activity intensity. There were no significant changes in physical activity patterns or in aerobic fitness. Estimated total LTPA (median 1376, range $128-3380 \mathrm{kcal}$ week21) was less than that recommended to improve aerobic fitness and/or slow progression of coronary artery disease. Sixteen subjects attended a median of 29 (range 1-46) AFL during LTPA; one way ANOVA showed these subjects worked at greater EEE (AFL, $\mathrm{n}=16,6.6$ (standard deviation 1.4) $v$ no-AFL, $\mathrm{n}=15,5.1$ (1.8) EEE kcal $\left.\min ^{-1}, \mathrm{p}=0.017\right)$.

Conclusion: Physical activity was stable, but patients' EEE appeared insufficient to improve aerobic fitness or slow progression of coronary artery disease. It was suggested that the promotion of LTPA and the availability of AFL classes should be reconsidered.

(Br J Sports Med 2005;39:el2) http://bjsm.bmjjournals.com/ cgi/content/full/39/3/el2 